\title{
Ultraviolet characterization of integrating spheres
}

\author{
Ping-Shine Shaw, ${ }^{\star}$ Zhigang Li, Uwe Arp, and Keith R. Lykke \\ Physics Laboratory, National Institute of Standards and Technology, Gaithersburg, Maryland 20899, USA \\ ${ }^{*}$ Corresponding author: shaw@nist.gov
}

Received 20 February 2007; accepted 26 March 2007;

posted 10 April 2007 (Doc. ID 80231); published 9 July 2007

\begin{abstract}
We have studied the performance of polytetrafluoroethylene integrating spheres in the ultraviolet (UV) region with wavelengths as short as $200 \mathrm{~nm}$. Two techniques were used for this study; first, the spectral throughput of an integrating sphere irradiated by a deuterium lamp was analyzed by a monochromator. Second, a UV laser beam was directed into an integrating sphere, and spectrally dispersed laser induced fluorescence was studied. Significant absorption and fluorescence features were observed in the UV region and attributed to the contamination in the integrating sphere. We demonstrate that integrating spheres are easily contaminated by environmental pollutants such as polycyclic aromatic hydrocarbons emitted from engine exhaust. Baking of the contaminated integrating sphere can reverse some but not all of the effects caused by contaminants. The implications for using integrating spheres for UV measurement are discussed.
\end{abstract}

OCIS codes: $120.3150,140.3610,300.2530,120.5630$.

\section{Introduction}

Integrating spheres play an indispensable role when it comes to diffusing and depolarizing radiation. They are widely used in radiometric applications because of their superior performance in transforming radiation to a nearly ideal Lambertian distribution despite variations in the conditions of the incident radiation. In the visible and near ultraviolet (UV) region, polytetrafluoroethylene (PTFE) has been proven to be the best material for integrating spheres because of high reflectivity and chemical inertness. Integrating spheres made with sintered PTFE are commercially available from many manufacturers.

For UV applications, however, concerns over the performance of diffusing materials, such as PTFE, were first raised three decades ago [1] when integrating spheres were used for spectral irradiance measurements of deuterium lamps. When integrating spheres were irradiated by UV radiation from deuterium lamps, spurious effects in the measured spectral irradiance were observed and attributed to fluorescence from diffusing materials induced by UV irradiation. A later study [2] confirmed the fluorescence effect from PTFE powder and suggested that contaminants in the powder were responsible for fluorescence.
Since then only a few studies on the UV reflectance and fluorescence of diffusing materials have been reported [3-7] and, to the best knowledge of the authors, no UV work on integrating spheres has been documented. In one study of PTFE as a diffuse reflectance material for space-borne remote sensing application $[3,4]$, contaminants were again shown to be responsible for the degradation in the reflectance of PTFE samples when irradiated with radiation from a Xe lamp. By using several chemical analysis techniques, the nature of the contaminants was identified only as volatile hydrocarbon impurities intrinsic to the commercial material. Furthermore, it was also shown that contaminants in PTFE samples can be cleaned by baking in vacuum although a clean PTFE sample can be contaminated again when exposed to aromatic hydrocarbons. Later, more studies reported measurements of the degradation in the hemispherical reflectance of PTFE material irradiated by $\mathrm{Xe}$ or $\mathrm{Hg}$ lamps [5-7] with no mention of its implication for PTFE integrating spheres.

The correlation between the hemispherical reflectance of PTFE material and the throughput of a PTFE integrating sphere is given by the following expression [8] 


$$
\boldsymbol{\Phi}_{\text {out }}=\boldsymbol{\Phi}_{\text {in }} \frac{r^{2} A_{2}\left[1-\left(A_{1}+A_{2}\right)\right]}{1-r\left[1-\left(A_{1}+A_{2}\right)\right]}
$$

where $\boldsymbol{\Phi}_{i n}$ and $\boldsymbol{\Phi}_{\text {out }}$ are the flux entering and exiting an integrating sphere, $A_{1}$ and $A_{2}$ are the ratio of the area of the entrance and exit port to the total inner surface area of the sphere, and $r$ is the hemispherical reflectance of the diffusing material. At high reflectance and small port area, as is the case for a typical integrating sphere, the throughput is highly sensitive to small variations in $r$. Given the reported vulnerability to degradation in the UV hemispherical reflectance of PTFE, it is expected that the degradation in the throughput of a PTFE integrating sphere will be much more pronounced. The instability of the UV throughput of an integrating sphere could result in large errors in long-term measurements.

In addition to throughput, fluorescence from an integrating sphere is even more challenging to UV measurement. Fluorescence, generally induced by incident radiation from a wavelength region to the blue, introduces spurious spectral effects and leads to measurement errors that are difficult to identify. A study of the UV properties of integrating spheres in both throughput and florescence will have important implications for radiometric measurements in the UV, such as source calibration, reflectance standards, and remote sensing [9].

Integrating spheres made with PTFE are formed by two major techniques. Most commercially available products are made with sintered PTFE and many laboratories, including the National Institute of Standards and Technology (NIST), also construct their own integrating spheres by pressing PTFE powder onto a spherical surface. Sintered PTFE integrating spheres are more structurally sound but concerns have been raised about the resin used to form the spherical shape [3]. Here, we report the characterization of both sintered and pressed PTFE integrating spheres in the UV to identify the nature of an integrating sphere's absorption and fluorescence properties using two techniques. First, the integrating sphere under study is irradiated by a deuterium lamp, and the relative spectral throughput of the integrating sphere is measured using a monochromator system. Second, the integrating sphere is irradiated with a beam from a tunable UV laser, and the laser induced fluorescence (LIF) is analyzed by a spectrometer. The former technique measures the absorption spectra and total fluorescence while the latter provides fluorescence spectra excited by a fixed wavelength. The combination of absorption and fluorescence spectra provides insight into the chemical nature of any contaminants responsible for fluorescence. Many commercially available sintered PTFE integrating spheres, along with home-made pressed PTFE integrating spheres, were measured using both techniques. We studied the role of contaminants for the observed absorption and fluorescence as well as the degradation of integrating spheres under UV irradiation. We also studied integrating spheres with controlled exposure to environmental pollutants, such as gasoline and diesel exhaust from engines. The effect of baking integrating spheres in both vacuum and air on contaminants is discussed. Detailed results and analysis as well as some recommendations on using integrating spheres for UV work are given.

\section{Experimental Setup}

In characterizing integrating spheres, two UV sources were used to probe the integrating sphere under study; a deuterium lamp and a tunable laser system. The deuterium lamp emits continuous radiation in the UV for the study of spectral throughput while the laser system provides several discrete wavelengths for LIF study. By using these two sources, we investigated both pressed and sintered PTFE integrating spheres, with the former made at NIST using PTFE powders and the latter from several different manufacturers. For better comparison, all of the spheres investigated were chosen with an inner diameter $\sim 3.8 \mathrm{~cm}$ and $\sim 1 \mathrm{~cm}$ diameter for both entrance and exit apertures. Pristine integrating spheres were also subject to different treatments and studied using both techniques. These treatments include baking of the integrating sphere, prolonged exposure to a deuterium lamp for stability study, and exposure to engine exhaust to assess the effect of environmental pollutions.

\section{A. Spectral Throughput Measurements}

The spectral throughput of an integrating sphere was measured by utilizing part of a UV irradiance calibration system at the Facility for Irradiance Calibration Using Synchrotrons (FICUS) at NIST $[10,11]$. As shown in Fig. 1, a deuterium lamp was placed at a typical distance of $30 \mathrm{~cm}$ from the entrance port of the integrating sphere under study. The spectral distribution of the exiting radiation from the integrating sphere was measured with a $0.25 \mathrm{~m}$ Czerny-Turner monochromator coupled to a temperature-stabilized photomultiplier tube. The bandwidth of the monochromator was set at $5 \mathrm{~nm}$ and the wavelength range was scanned between 200 and $400 \mathrm{~nm}$ for this work. Order-sorting filters were not necessary for this

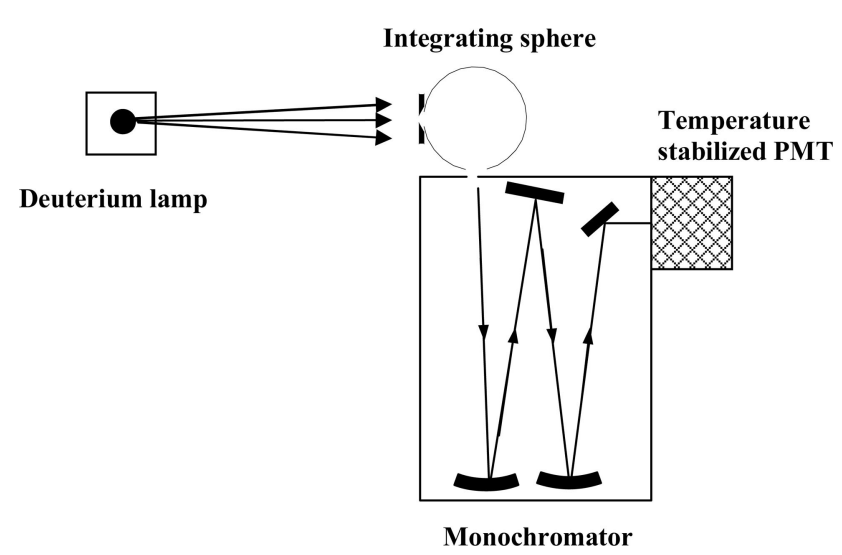

Fig. 1. Schematic diagram of the experimental setup for measurement of integrating sphere spectral throughput. 


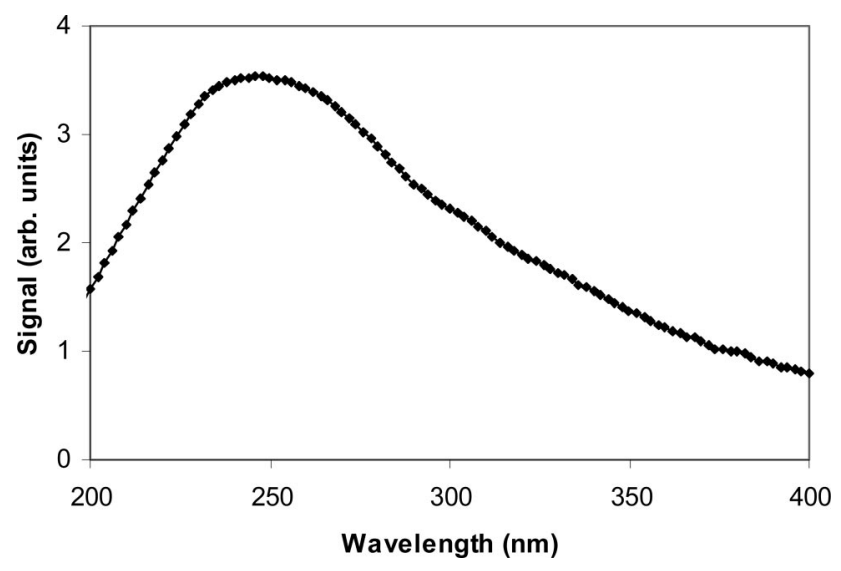

Fig. 2. Spectral output with the deuterium lamp irradiating the monochromator entrance directly. This signal was used to normalize spectral outputs measured with integrating spheres to obtain the relative throughput of the integrating spheres as explained in the text.

range since wavelengths shorter than $200 \mathrm{~nm}$ were eliminated by air and window absorption.

The measured spectral distribution using the above setup consists of several spectral effects, such as the output of the deuterium lamp, the throughput of the integrating sphere, the throughput of the monochromator, and the responsivity of the photomultiplier tube. To isolate the throughput of the integrating sphere, a separate measurement was performed by removing the integrating sphere and placing the deuterium lamp directly in front of the entrance slit of the monochromator. The resulting spectral output, as shown in Fig. 2, was used to normalize all spectral distributions measured with an integrating sphere. While this normalization process does not yield absolute throughput of the integrating sphere under study at a specific wavelength, the normalized spectral distribution is proportional to the spectral throughput of the integrating sphere by a constant throughout the wavelength region of measurements. Absorption structures in the integrating spheres can be easily identified from normalized spectral distributions.

\section{B. Laser Induced Fluorescence Measurements}

Tunable lasers at the NIST facility for Spectral Irradiance and Radiance Responsivity Calibrations using Uniform Sources [12] were used for the study of LIF from integrating spheres. The tunable laser, with a range of wavelength from 200 to $400 \mathrm{~nm}$ for this work, was generated by frequency doubling, tripling, and quadrupling of a quasi-continuous wave (cw) mode-locked Ti:sapphire laser. The quasi-cw output, at $76 \mathrm{MHz}$ and $2 \mathrm{ps}$ duration, was in the $\mathrm{mW}$ power range. The wavelength uncertainty was less than $0.01 \mathrm{~nm}$.

The setup used to measure fluorescence from an integrating sphere is very similar to the setup used to measure the spectral throughput using a deuterium lamp discussed previously except that the output radiation from the integrating sphere was measured by a

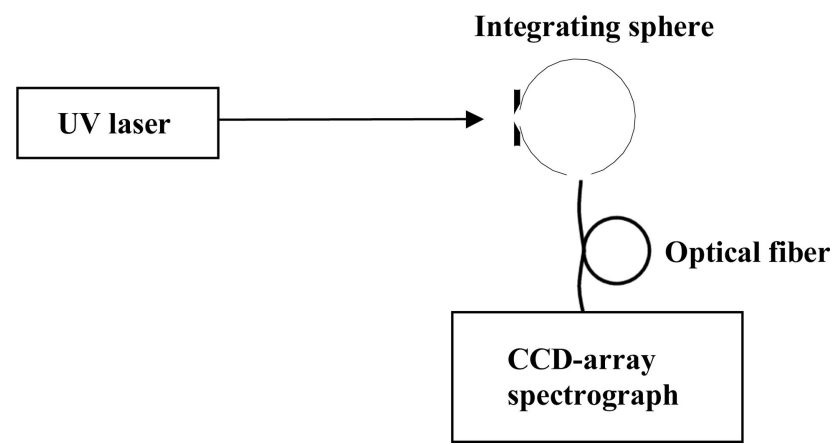

Fig. 3. Schematic diagram of the experimental setup for the LIF measurements on integrating spheres.

commercial charge coupled device (CCD)-array spectrograph instead of the monochromator as shown in Fig. 3. Because of the high input laser power irradiating the integrating sphere, the CCD-array spectrograph was used to achieve a wider spectral range (from 200 to $800 \mathrm{~nm})$, higher resolution $(\sim 3 \mathrm{~nm}$ in bandwidth), and shorter acquisition time. An optical fiber directed the radiation from the exit port of the integrating sphere to the entrance port of the spectrograph. Inside the spectrograph, dispersed radiation was detected by a two-dimensional cooled $\left(-10^{\circ} \mathrm{C}\right)$ CCD array with a total of $1024 \times 128$ pixels. The output of the array detector was binned along the column with 128 pixels, and the resolution of the instrument was 15 bits.

The measured spectral distribution using the LIF setup with fixed wavelength laser excitation consists of fluorescence from the integrating sphere and the primary exciting laser line on top of two slowly decaying wings around the primary laser peak (i.e., line spread function) contributed by scattered stray light inside the spectrograph $[11,13,14]$. Shown in Fig. 4 is a typical line spread function measured with a $360 \mathrm{~nm}$ laser beam. For better comparison between different measurements, all spectral distributions are normalized to the peak at the wavelength of the excitation laser.

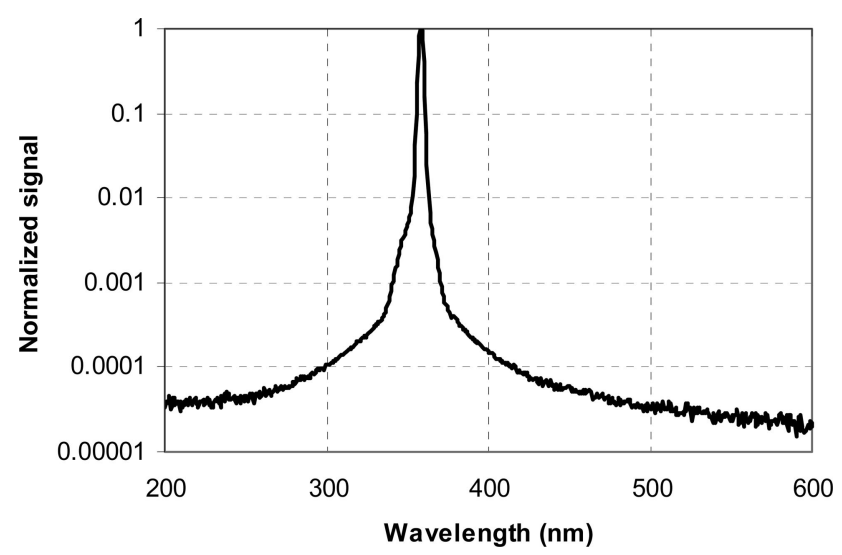

Fig. 4. Typical line spread function of the spectrograph with the laser wavelength of $360 \mathrm{~nm}$. 


\section{Results}

To have a general evaluation on the impact of absorption and fluorescent effects of integrating spheres on the UV radiometry, we characterized a number of newly acquired commercial integrating spheres from several manufacturers. For comparison, we also studied some commercial integrating spheres that have been extensively used in our laboratory. Pressed integrating spheres were not commercially available and were packed at NIST using PTFE powders of Halon [15]. The effect of baking integrating spheres was studied at length. We also measured integrating spheres that were exposed to environmental contamination to shed light on the causes of UV absorption and fluorescence. Finally, we discuss the temporal stability of integrating spheres.

\section{A. Pristine Integrating Spheres}

Shown in Fig. 5 are the results of spectral throughput measurements performed on several sintered and pressed integrating spheres. Note that all throughput curves were normalized with the throughput at $400 \mathrm{~nm}$ to account for the variation in the integrating sphere throughput owing to differences in sphere geometry. All curves in Fig. 5 display a general trend of gradual decrease in throughput toward shorter wavelengths in accordance with the reported decrease in reflectivity from PTFE plaques [2,9]. In addition to the gradual decay, all integrating spheres show the same two-peak-absorption structure below $300 \mathrm{~nm}$. Note that this structure is very similar to the measured reflectance factor of sintered PTFE samples reported previously [9]. For wavelengths above $300 \mathrm{~nm}$, the throughput is generally smooth, but some integrating spheres exhibited a broad peak. Of all integrating spheres shown in Fig. 5, the pressed integrating sphere has the smoothest spectral throughput with the absorption structure barely distinguishable, in-

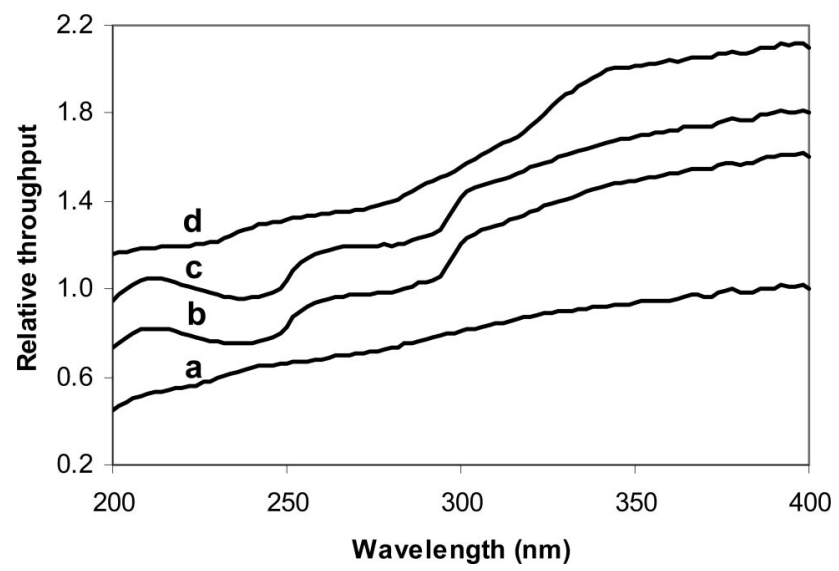

Fig. 5. Relative throughput measured with (a) a freshly pressed PTFE integrating sphere, and (b)-(d) commercial sintered PTFE integrating spheres with (b) and (c) new from manufacturers when measured, while (d) had been used for UV radiometry prior to this measurement. All curves are normalized at $400 \mathrm{~nm}$ to 1 . For easy viewing, there is an offset to each curve. The offsets are 0 for (a), 0.6 for (b), 0.8 for (c), and 1.1 for (d).

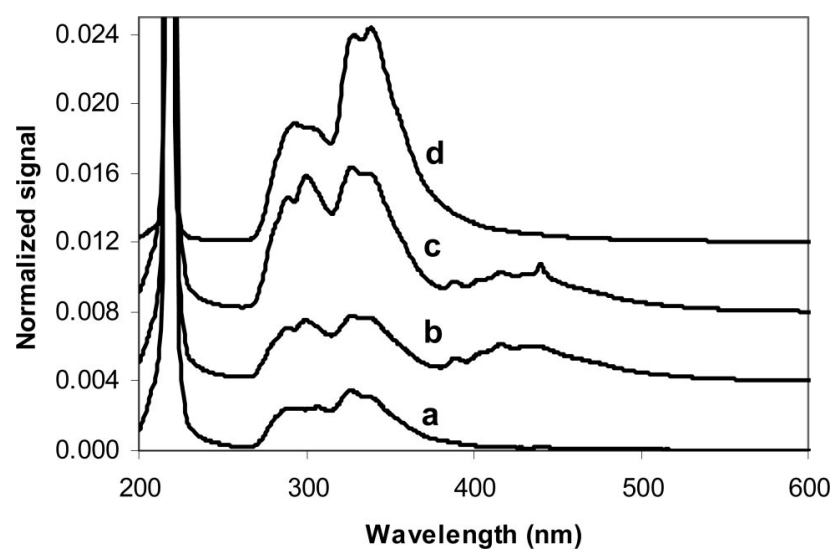

Fig. 6. LIF measurements of (a) a pressed PTFE integrating sphere and (b)-(d) commercial sintered PTFE integrating spheres with the excitation laser tuned at $220 \mathrm{~nm}$. All curves are normalized to 1 at the laser excitation wavelength of $220 \mathrm{~nm}$. For easy viewing, there is an offset to each curve. The offsets are 0 for (a), 0.004 for (b), 0.008 for (c), and 0.012 for (d).

dicating that the absorption structure is contributed to by contaminants and not an intrinsic property of the PTFE material. In general, we found that pressed PTFE integrating spheres are much less infected with these absorption bands as well as fluorescence, as will be discussed later. This is again in accordance with previous observations [9].

To find the fluorescence properties of the integrating spheres under this study, we conducted LIF measurements for several integrating spheres used in Fig. 5. The measured fluorescence spectra with the excitation laser wavelength set at $220 \mathrm{~nm}$ are shown in Fig. 6. As in the case of the throughput spectra, the fluorescence spectra resemble each other in the wavelength region from 250 to $400 \mathrm{~nm}$. In addition, two sintered PTFE integrating spheres exhibit a broad fluorescence band between 400 and $500 \mathrm{~nm}$. In terms

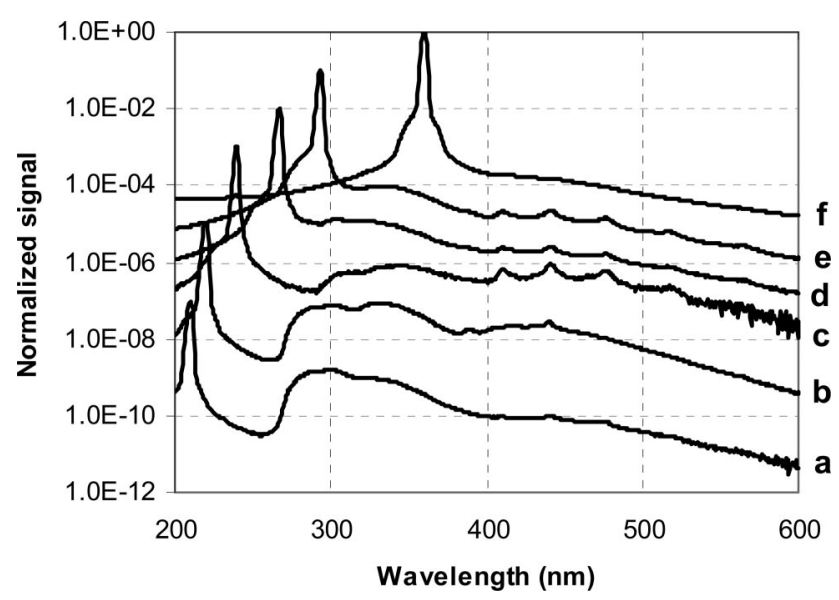

Fig. 7. LIF measurements of a commercial sintered PTFE integrating sphere with the excitation laser tuned at (a) 210, (b) 220 , (c) 240, (d) 266, (e) 293, and (f) $360 \mathrm{~nm}$. For easy viewing, the peak of each curve at the wavelength of the excitation laser is normalized to a different value. The values are $10^{-7}$ for (a), $10^{-5}$ for (b), $10^{-3}$ for (c), $10^{-2}$ for (d), $10^{-1}$ for (e), and 1 for (f). 
of fluorescence yield, we again found that the pressed PTFE integrating sphere with the least visible absorption band in throughput has the smallest fluorescent yield over other sintered PTFE integrating spheres.

The fluorescence spectra of integrating spheres are also strongly dependent on the wavelength of the exciting laser beam as depicted in Fig. 7 for a sintered PTFE integrating sphere. The fluorescence between 250 and $400 \mathrm{~nm}$ decreases as the wavelength of the laser increases. On the other hand, the fluorescence band between 400 and $500 \mathrm{~nm}$ shows a distinct structure with laser wavelengths of 240,266 , and $360 \mathrm{~nm}$.
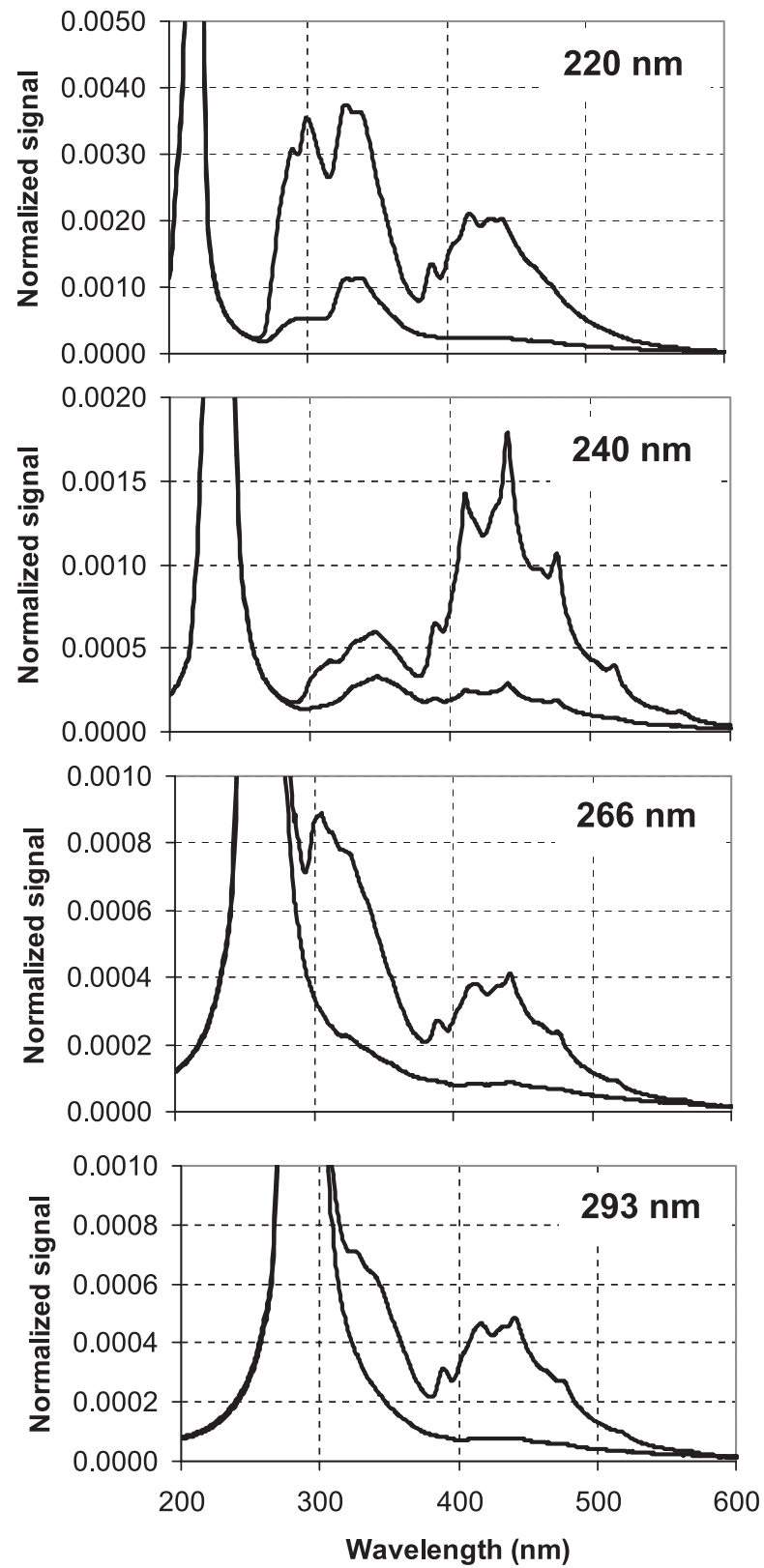

Fig. 8. LIF measurements of a sintered PTFE integrating sphere before and after baking the integrating sphere $40 \mathrm{~h}$ in vacuum. For each graph, the top curves are before baking and the bottom curves are after baking. All curves are normalized to 1 at the corresponding laser excitation wavelengths.
These fluorescence peaks have a nearly equal spacing of $1700 \mathrm{~cm}^{-1}$, indicating vibrational progression from different vibrational transitions.

\section{B. Effects of Baking}

Baking of integrating spheres in vacuum has been previously suggested as a way to remove contaminants [3]. With the characterization techniques used for this study, we were able to determine the effectiveness of baking integrating spheres both in vacuum and in air. We found that the effects of baking are easier to quantify using the LIF measurements. Shown in Fig. 8 is the LIF measurement using several exciting laser wavelengths performed on a sintered PTFE integrating sphere before and after baking in vacuum for $40 \mathrm{~h}$ at $90{ }^{\circ} \mathrm{C}$. It is clear from Fig. 8 that the fluorescence yield is much reduced after baking, but not eliminated for all wavelengths. Notice also that the reduction of fluorescence between 250 and $400 \mathrm{~nm}$ is much less than that between 400 and $500 \mathrm{~nm}$. Overall, the remnants of fluorescence after baking still resemble the spectra before baking. Our tests show that longer baking time in vacuum does not completely eliminate the fluorescence. This fact suggests that the contaminants in an integrating sphere are somewhat volatile and baking can remove some, but not all, contaminants.

For even longer laser wavelengths than those shown in Fig. 8, the fluorescence is relatively small and, by itself, cannot be easily separated from the line spread function of the monochromator. Nevertheless, the fluorescence can be readily identified by comparing the fluorescence spectra of integrating spheres before and after baking. Figure 9 shows such a comparison for a laser wavelength of $360 \mathrm{~nm}$. The fluorescence between 400 and $500 \mathrm{~nm}$ before the bake is evident.

Similar results, shown in Fig. 10, were obtained for baking a pressed PTFE integrating sphere. While we observed no discernible fluorescence between 400 and $500 \mathrm{~nm}$, the reduction of fluorescence between 250

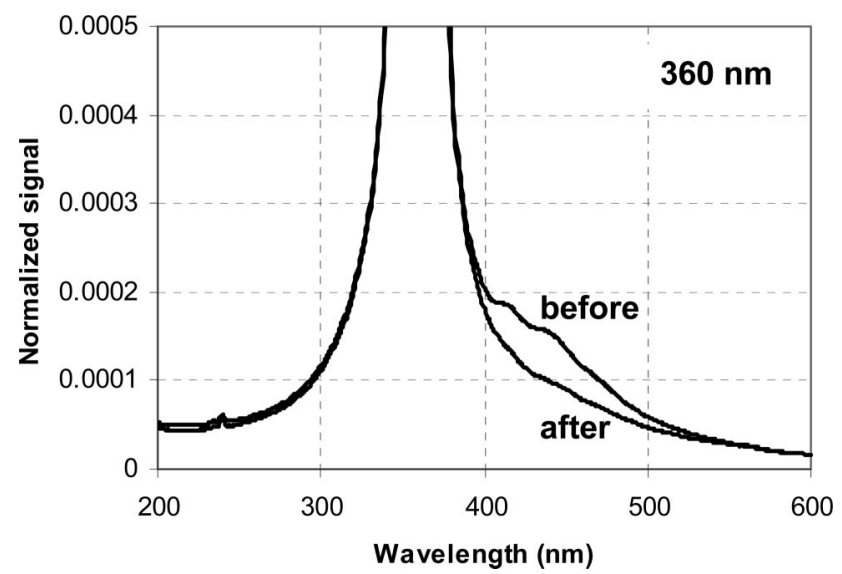

Fig. 9. LIF measurements of a sintered PTFE integrating sphere before and after baking the integrating sphere $40 \mathrm{~h}$ in vacuum. The top curve is before baking and the bottom curve is after baking. 

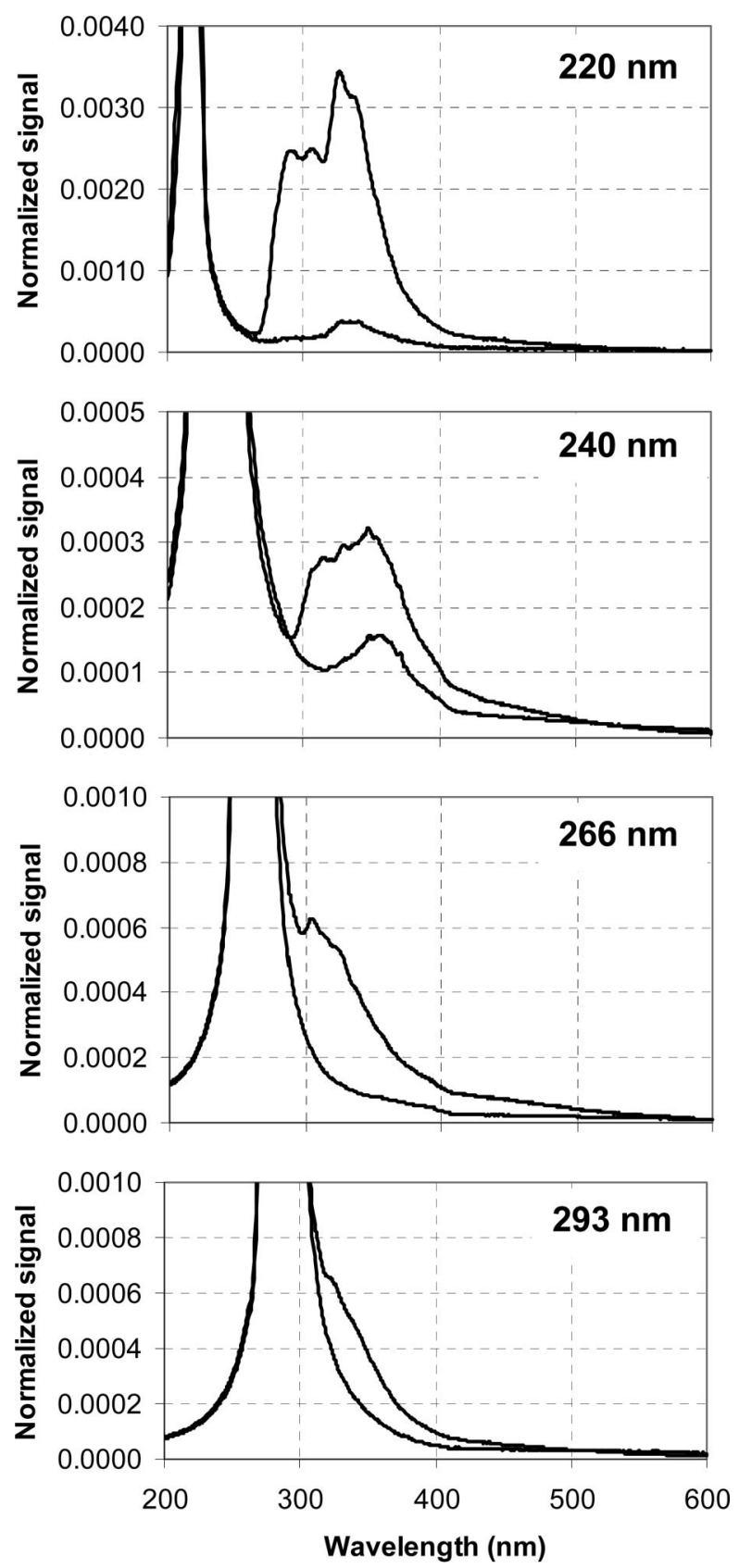

Fig. 10. LIF measurements of a pressed PTFE integrating sphere before and after baking the integrating sphere $40 \mathrm{~h}$ in vacuum. For each graph, the top curves are before baking and the bottom curves are after baking. All curves are normalized to 1 at the corresponding laser excitation wavelengths.

and $400 \mathrm{~nm}$ due to baking is very similar to that of a sintered integrating sphere shown in Fig. 8.

It is also of interest to investigate the throughput change of an integrating sphere after baking. Shown in Fig. 11 is the throughput of two sintered PTFE integrating spheres and a pressed PTFE integrating sphere before and after baking in vacuum and in air. It is evident that baking tends to smooth the throughput spectra from the absorption structure below $300 \mathrm{~nm}$. Baking also improves the throughput in the short wavelength range; while in some regions on the
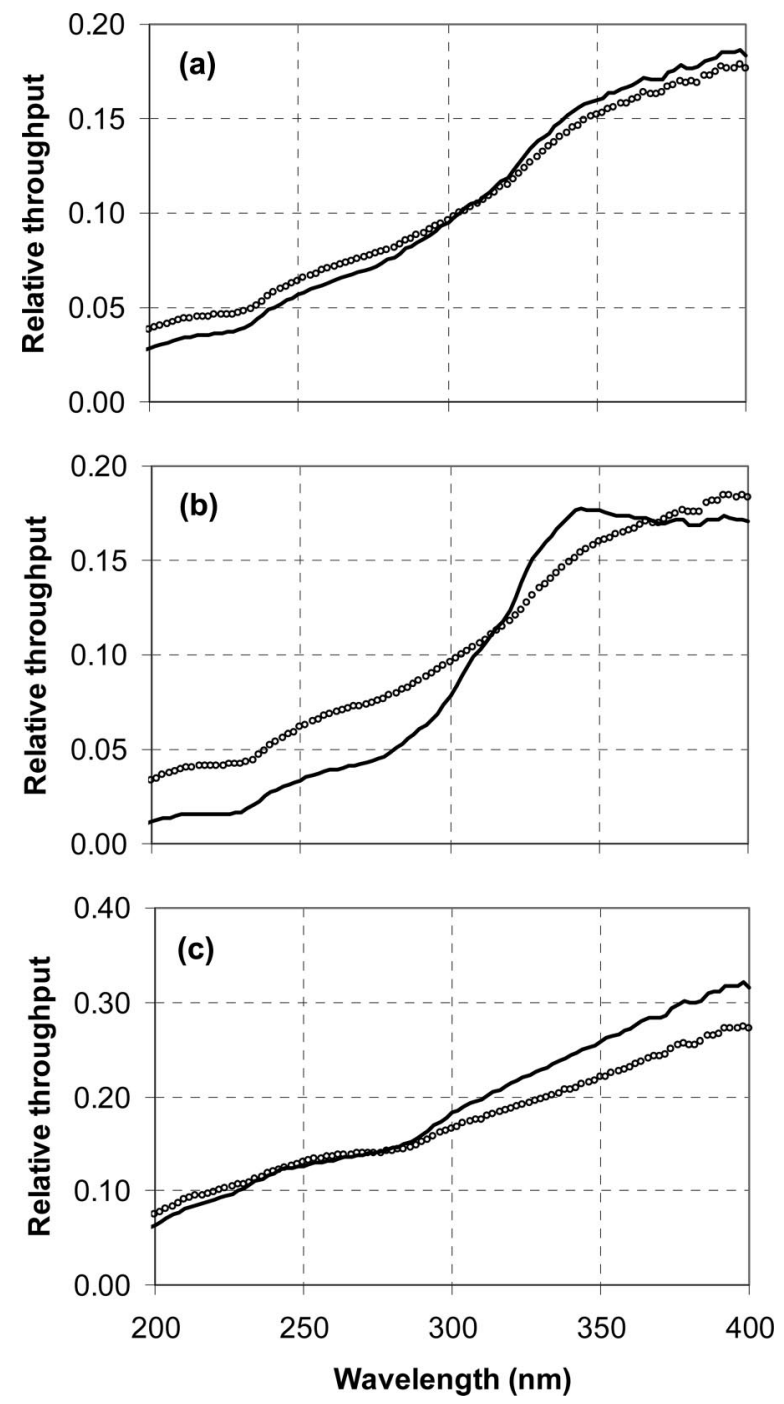

Fig. 11. Relative throughput measurements of (a) a sintered PTFE integrating sphere before and after $65 \mathrm{~h}$ vacuum bake, (b) a sintered PTFE integrating sphere before and after $24 \mathrm{~h}$ air bake, and (c) a pressed PTFE integrating sphere before and after $40 \mathrm{~h}$ vacuum bake. Solid lines are before the bake, and open circles are after the bake.

longer wavelength side, throughput decreases. In view of the changes of the fluorescence spectra from baking, we can interpret this phenomenon as follows: the absorption structure below $300 \mathrm{~nm}$ is caused by the absorption of UV radiation from contaminants inside the integrating sphere. As the integrating sphere is baked, some of the contaminants leave the integrating sphere, which leads to improved UV throughput with reduced absorption structure. On the other hand, less contaminants cause decreased fluorescence, which predominantly fluoresce with wavelengths longer than $300 \mathrm{~nm}$.

Finally, we studied and compared the effects of baking an integrating sphere in vacuum and in air, which as a practical matter could be done much easier and less costly. For this comparison, we packed two integrating spheres with the same batch of Halon PTFE powder under the same procedure. One inte- 


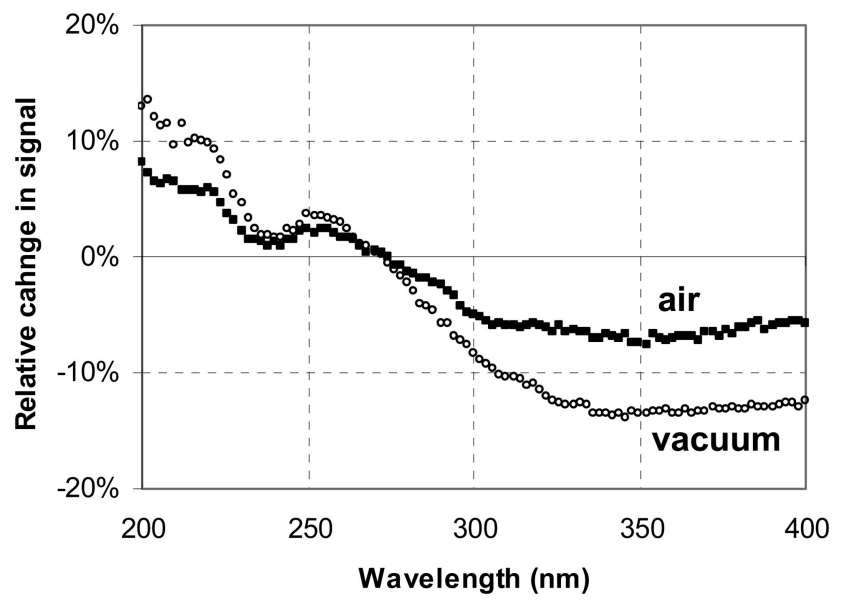

Fig. 12. Relative changes in throughput for pressed integrating spheres after $24 \mathrm{~h}$ vacuum bake and after $105 \mathrm{~h}$ air bake.

grating sphere was baked under vacuum at $90{ }^{\circ} \mathrm{C}$ with a base pressure of $10^{-3} \mathrm{~Pa}$; while the other was baked in an oven in air, also at $90{ }^{\circ} \mathrm{C}$. The changes in the spectral throughput for both integrating spheres are shown in Fig. 12 for $24 \mathrm{~h}$ vacuum baking and $105 \mathrm{~h}$ baking in air. Both spectra show increased throughput for the short wavelength side and decreased throughput for the longer wavelength side. It is clear from Fig. 12 that baking in vacuum is much more effective than in air. In Fig. 13, we show the change for throughput at two wavelengths, one long $(396 \mathrm{~nm})$ and one short $(206 \mathrm{~nm})$, as a function of the baking time in air. Even after more than 4 days of baking, a plateau was not reached.

\section{Effects of Exposure to Environmental Pollutants}

The stability of an integrating sphere is critical when used in a calibration facility, such as FICUS. In calibrating deuterium lamps at FICUS [11], we observed that an integrating sphere can have dramatic changes in its throughput over a few days. The cause of such a change was traced to diesel truck fumes leaking into the room where the measurement was

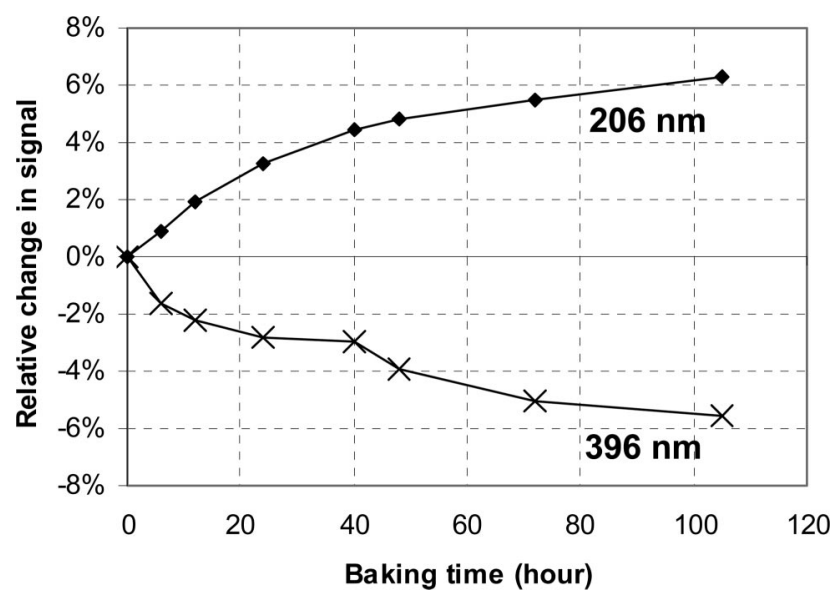

Fig. 13. Relative change in throughput for pressed integrating spheres as a function of baking time in air for 206 and $396 \mathrm{~nm}$. performed. Since exhaust from vehicles is a common source of aromatic compounds and it was reported previously that PTFE integrating spheres are susceptible to contamination by hydrocarbon aromatic compounds [3], it is of interest to examine the behavior of integrating spheres when exposed to such environmental pollutants.

This simple but effective experiment started with a fresh well-baked sintered PTFE integrating sphere with its UV fluorescence and absorption measured. The integrating sphere was then placed near the tail pipe of a car or a truck with its engine running. After several seconds of exposure, the integrating sphere was removed and taken into our laboratories for both throughput and LIF measurements again. We repeated the experiment for both a gasoline-powered car and a diesel-powered truck, and the results are shown in Figs. 14 and 15. Note that these measurements were not performed under well controlled exposures and the results provide a clear qualitative characterization on the behavior of integrating spheres when exposed to pollutants.

For the LIF measurements in Fig. 14, exposure to gasoline and diesel exhaust increases the fluorescence, remarkably, in exactly the same spectral regions where the integrating spheres fluoresce before the exposure to exhaust. In particular, the increase in fluorescence spectra from diesel exhaust exposure between 250 and $400 \mathrm{~nm}$ closely resembles that observed for pristine integrating spheres (shown in
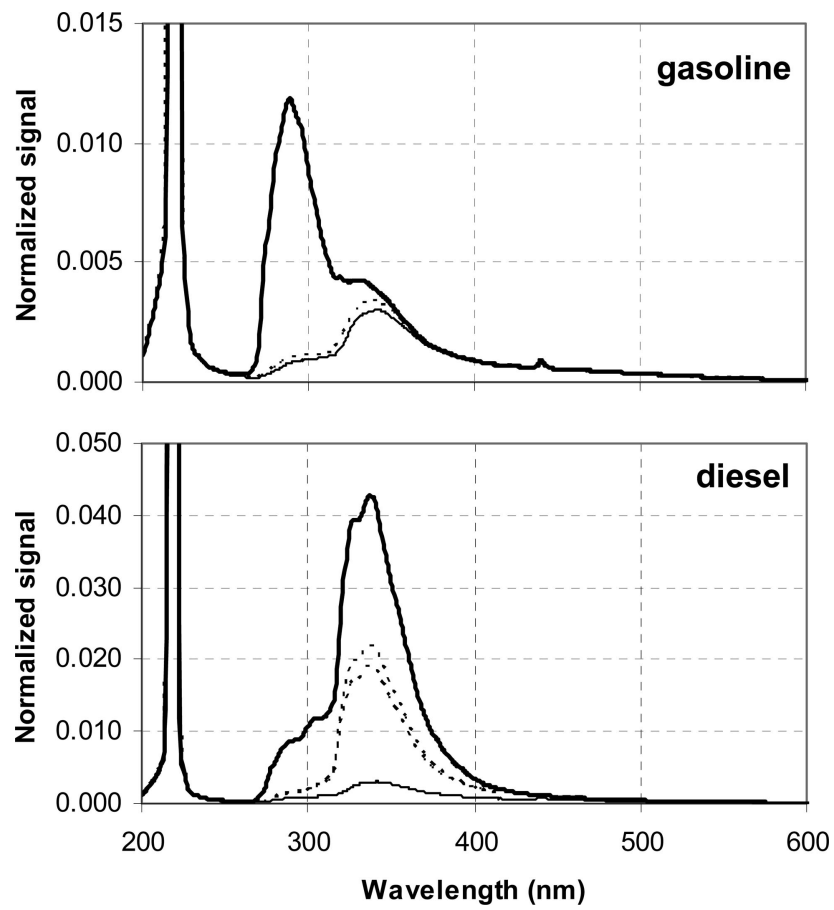

Fig. 14. LIF measurements of a sintered PTFE integrating sphere exposed to exhaust from a gasoline engine and from a diesel engine. Thin solid lines are before the exposure, thick solid lines are after the exposure. For gasoline exposure, dotted line is $19 \mathrm{~h}$ of baking in vacuum after exposure. For diesel exposure, dotted lines are baking for both 19 and $68 \mathrm{~h}$. 


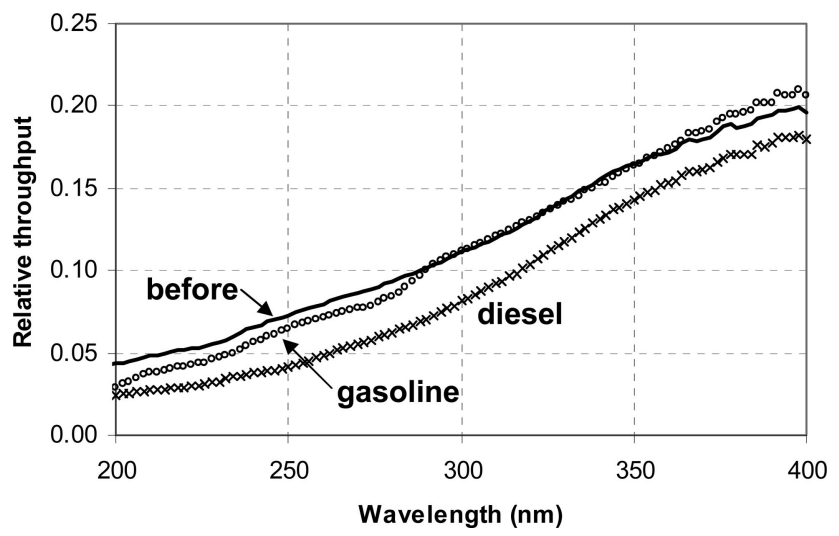

Fig. 15. Relative throughput measurements of a sintered PTFE integrating sphere before exposure, after exposure to gasoline engine exhaust, and after exposure to diesel engine exhaust.

Fig. 10). As one might expect, the diesel exhaust exposure caused a much stronger increase in fluorescence than the gasoline exhaust.

Figure 14 also shows the results of baking the exposed integrating sphere to remove the contaminants. For gasoline exhaust, after $19 \mathrm{~h}$ of baking in vacuum at $90^{\circ} \mathrm{C}$, the fluorescence spectrum was almost fully restored to its original spectra before exposure to the exhaust. For diesel exhaust, the fluorescence was only partially reduced even after more than $80 \mathrm{~h}$ of baking in vacuum. Other cleaning techniques we tried, such as the use of various solvents, did not restore the integrating sphere to its state before exposure. Currently, we are still experimenting with other options to rid the integrating spheres of contaminants.

For the throughput measurements on the exposed integrating spheres shown in Fig. 15, the diesel exposure caused a substantial decrease in throughput over the whole wavelength region of our measurement, indicating the presence of a large amount of contaminants. As in the case of the LIF measurement, baking partially restored the throughput to that before the exposure. Lastly, exposure to gasoline exhaust resulted in the buildup of the two-peakabsorption structure in the shorter wavelengths we discussed previously. For the longer wavelengths, there is a slight increase in the throughput. This is in contrast to the observed decrease in throughput after baking an integrating sphere discussed previously.

\section{Ultraviolet Stability}

In radiometric applications aiming for the highest measurement accuracy, the stability of an optical element, such as an integrating sphere, is an essential property [5]. Given the vulnerability of PTFE integrating spheres to contamination as discussed above, it is not surprising that the stability of an integrating sphere is primarily influenced by the level of contamination. We have performed several preliminary tests to check the stability in the throughput of an integrating sphere under UV irradiation from a deuterium lamp.
Our preliminary results show that, in general, the throughput of an integrating sphere with relatively small absorption and fluorescence features, such as a baked integrating sphere, is stable to within a few percent in the UV after a few days of continuous irradiation from a deuterium lamp. On the other hand, the throughput of an integrating sphere with large absorption and fluorescence features varies as much as $10 \%$ under the same UV irradiation even for a few hours. The exact change in throughput varies from one sphere to another. At this time, it is not clear if the instability is caused by UV radiation interacting with the contaminants or a continued buildup of contaminants from the surrounding environment. Further experiments are under way to shed light on the mechanism for such an instability.

\section{Discussion and Conclusions}

As discussed previously, all PTFE integrating spheres studied for this work showed a consistent but varying degree of absorption and fluorescence features in the UV, and moderate baking can reduce these features. This indicates that the observed fluorescence and the associated absorption in the UV are not intrinsic to the PTFE material. Contamination of integrating spheres-by somewhat volatile impurities either during the manufacturing process or exposure to contaminating sources afterward-is mostly responsible for the observed effects. This is supported by a previous study where the existence of impurities, identified as organic hydrocarbons, was confirmed [3]. Furthermore, it was shown that PTFE can absorb aromatic hydrocarbons of toluene and naphthalene after exposure to these compounds [3]. Toluene and naphthalene belong to a class of the polycyclic aromatic hydrocarbons (PAHs), and it is worthwhile to compare our results with that of PAHs in general.

Known for chemical stability from their conjugated $\pi$-electron systems, PAHs typically exhibit strong absorption and fluorescence spectra in the UV [16] as a result of the transition of an electron between $\pi$-bonding to $\pi^{*}$-antibonding orbitals $[17,18]$. The transition normally involves a ground vibrational level of a ground singlet electronic state to a vibrationally excited level of an excited singlet electronic state. The resulting fluorescence is typically redshifted from the absorption because of transitions to higher vibrational levels of the ground electronic state. Because the vibrational level spacings in ground and excited electronic states are usually similar, the fluorescence spectrum is often virtually the "mirror image" of the absorption spectrum [19]. In our observation of integrating spheres, the persistent absorption band between 200 and $300 \mathrm{~nm}$ and the fluorescence band between 250 and $400 \mathrm{~nm}$ fits very well with that of PAH impurities. For some sintered PTFE integrating spheres, the additional fluorescence band observed between 400 and $500 \mathrm{~nm}$ (see Fig. 8) contains a clear vibrational structure $\left(\sim 1700 \mathrm{~cm}^{-1}\right.$ spacing) and is caused by different PAH impurities. Further support 
for $\mathrm{PAH}$ impurities is given by the close resemblance of the fluorescence spectra from 250 to $400 \mathrm{~nm}$ between pristine integrating spheres and integrating spheres exposed to engine exhaust, which is known to contain a large number of PAHs.

While the exact chemical compounds of the PAH impurities cannot be easily identified by absorption and fluorescence spectra alone, one can still obtain information about the chemical nature of the impurities from the measured spectra using properties that are generally true for PAHs. In general, as the number of conjugated aromatic rings increases, the UV absorption maxima are shifted to longer wavelengths and exhibit a greater complexity of its absorption spectrum [20]. This explains the low response-for example, at $254 \mathrm{~nm}$-for PAHs with six or more rings [21]. Similarly for fluorescence, the spectrum also redshifts as the size of the conjugated aromatic ring system increases. The redshifting of the absorption and fluorescence spectrum can be explained as a particle (electron) in a box ( $\pi$-conjugated rings) modelthe larger the box, the more closely spaced the levels, and the longer the wavelength of absorption and emission. In the case of "linear" PAHs, such as benzene (one ring), naphthalene (two ring), anthracene (three ring), tetracene (four ring), and pentacene (five ring), a comparison between published fluorescence spectra [22] and our results shows that the measured fluorescence spectrum below $400 \mathrm{~nm}$ is caused by $\mathrm{PAHs}$ with one to three rings, while fluorescence between 400 and $500 \mathrm{~nm}$ is caused by three or four ring PAHs. The lack of fluorescence in the longer wavelength up to $800 \mathrm{~nm}$ indicates that only PAHs with a small number of conjugated aromatic rings were present in the integrating spheres we studied as impurities.

The measurements on integrating spheres exposed to engine exhaust also shed light on the nature and the origin of the impurities. Emission from vehicle exhaust contains a variety of $\mathrm{PAH}$ compounds and their derivatives depending on such conditions as the fuel type and the degree of incomplete combustion of fuel [17]. The emitted PAHs can be in the form of gas phase, or the PAH can be adsorbed or encapsulated in particulates. In general, diesel exhaust contains more particulates than gasoline exhaust. These particulates-diesel particulate matter (DPM)-include diesel soot and aerosols, such as ash particulates, metallic abrasion particles, sulfates, and silicates. Our LIF measurements on integrating spheres with exhaust exposure show that both gasoline and diesel exhaust deposit PAHs with a low number of aromatic rings, giving the similar fluorescence spectra from the pristine spheres that was identified above as $\mathrm{PAH}$ with one to three aromatic rings. The fact that diesel exhaust exposure induced a fluorescence peak in the slightly longer wavelength than gasoline exhaust exposure suggests that contaminants with higher ring-number PAHs resulted from diesel exhaust exposure. In the throughput measurements, as in LIF measurements, exposure to gasoline ex- haust caused similar absorption features for the pristine integrating spheres. However, diesel exposure caused a broad structureless absorption. This is most likely due to the deposition of DPM in the sphere that degraded the overall reflectance of PTFE. It is of interest to note that, in common environmental studies, PTFE membrane filters are preferred for the collection of particulates because of their high collection efficiency and inertness [17]. Finally, the fact that baking can restore an integrating sphere exposed to gasoline exhaust is in accordance with the volatility of some lighter $\mathrm{PAH}$ compounds, such as phenanthrene or anthracene, due to thermal desorption. On the other hand, it is possible that the difficulty in removing contaminants from a diesel exposed integrating sphere by baking is because either the PAHs involved have a much lower vapor pressure or the PAHs were trapped in the particulates, which are impossible to bake out at these low temperatures $\left(90^{\circ} \mathrm{C}\right)$.

Given the important role of PAHs as contaminants, there are two possible sources for PAH contamination: (1) the process of manufacturing the integrating sphere, such as the use of resin, and (2) the exposure of integrating spheres to an environment that contains PAHs. As shown above, engine exhaust in the environment will cause significant contamination that affects the performance of an integrating sphere in the UV. Even in normal air, suspended atmospheric particulate matter that in many cases contains a large number of different PAHs could also cause contamination. This clearly suggests that integrating spheres should be protected from contaminated air where possible to ensure longer stability in the UV. Once contaminated with PAH compounds, the PAHs can react in the air or undergo photoreaction with UV irradiation causing more instability. This was observed in our stability measurements where a contaminated integrating sphere tends to have much lower UV stability than a baked integrating sphere with less contamination.

From the standpoint of practical UV applications of integrating spheres, the absorption, fluorescence, and stability are of great concern for accurate measurements. The absorption in the UV causes lower throughput and to some extent affects the uniformity of the exiting radiation from an integrating sphere. Fluorescence is more challenging because it alters the spectral correlation between the input and output radiation. One way to correct this effect for an integrating sphere and monochromator system is to treat the fluorescence as part of the monochromator line spread function. With the combined system completely characterized using a tunable laser, contributions from fluorescence and scattering can be mathematically alleviated from the output spectra by using a stray light reduction algorithm as was previously demonstrated [11]. While the absorption and fluorescence problems can be solved in principle, the issue of instability caused by contamination from both the laboratory and field environment is more difficult to resolve. Baking and limiting exposure to 
environmental contaminants can help with stability. More research on the UV behavior of integrating spheres is currently underway to identify the molecules responsible for the contamination, find ways to clean the contaminants, and protect integrating spheres from further contamination.

In summary, we have used two techniques to characterize PTFE integrating spheres in the UV: measuring both the UV throughput spectrum using a deuterium lamp and the fluorescence spectrum induced by a tunable UV laser. All of the integrating spheres studied show significant absorption and fluorescence features. These features, more pronounced for sintered integrating spheres than pressed ones, are remarkably similar for all of the integrating spheres we studied and are consistent with PAH contaminants. Baking for long periods of time in vacuum can reduce the level of contaminants but does not completely eliminate them. In addition, fluorescence induced by the UV laser provided important clues for identifying the chemical nature of contaminants. $\mathrm{PAH}$ contaminants from the environment are the main suspect that deteriorate the performance of PTFE integrating spheres.

The authors thank Yuqin Zong for lending us the fiber optic spectrograph, and Robert Saunders for packing integrating spheres. We are also grateful to Howard Yoon and Albert Parr for their discussions and support of this project.

\section{References}

1. R. D. Saunders and W. R. Ott, "Spectral irradiance measurements: effect of UV-produced fluorescence in integrating spheres," Appl. Opt. 15, 827-827 (1976).

2. V. R. Weidner and J. J. Hsia, "Reflection properties of pressed polytetrafluoroethylene powder," J. Opt. Soc. Am. 71, 856-861 (1981).

3. A. E. Stiegman, C. J. Bruegge, and A. W. Springsteen, "Ultraviolet stability and contamination analysis of Spectralon diffuse reflectance material," Opt. Eng. 32, 799-804 (1993).

4. C. J. Bruegge, A. E. Stiegman, R. A. Rainen, and A. W. Springsteen, "Use of Spectralon as a diffuse reflectance standard for in-flight calibration of earth-orbiting sensors," Opt. Eng. 32, 805-814 (1993).

5. D. R. Gibbs, F. J. Duncan, R. P. Lambe, and T. M. Goodman, "Ageing of materials under intense UV radiation," Metrologia 32, 601-607 (1995/1996).

6. P. R. Spyak and C. Lansard, "Reflectance properties of pressed
Algoflon F6: a replacement reflectance-standard material for Halon,” Appl. Opt. 36, 2963-2970 (1997).

7. W. Möller, K.-P. Nikolaus, and A. Höpe, "Degradation of the diffuse reflectance of Spectralon under low-level irradiation," Metrologia 40, S212-S215 (2003).

8. H. J. Kostkowski, Reliable Spectroradiometry (Spectroradiometry Consulting, 1997), Appendix B.

9. P. Y. Barnes, M. E. Nadal, and E. A. Early, "Reflectance standards at ultraviolet wavelength," Proc. SPIE 3818, 9-14 (1999).

10. P. S. Shaw, U. Arp, H. Y. Yoon, R. D. Saunders, A. C. Parr, and K. R. Lykke, "A SURF beamline for synchrotron source-based absolute radiometry," Metrologia 40, S124-S127 (2003).

11. P. S. Shaw, U. Arp, R. D. Saunders, D. J. Shin, H. W. Yoon, C. E. Gibson, Z. Li, A. C. Parr, and K. R. Lykke, "Synchrotron radiation based irradiance calibration from $200 \mathrm{~nm}$ to $400 \mathrm{~nm}$ at SURF III," Appl. Opt. 46, 25-35 (2007).

12. S. W. Brown, G. P. Eppeldauer, and K. R. Lykke, "Facility for spectral irradiance and radiance responsivity calibrations using uniform sources," Appl. Opt. 45, 8218-8237 (2006).

13. S. W. Brown, B. C. Johnson, M. E. Feinholz, M. A. Yarbrough, S. J. Flora, K. R. Lykke, and D. K. Clark, "Stray-light correction algorithm for spectrographs," Metrologia 40, S81-S84 (2003).

14. Y. Zong, S. W. Brown, B. C. Johnson, K. R. Lykke, and Y. Ohno, "Simple spectral stray light correction method for array spectroradiometers," Appl. Opt. 45, 1111-1119 (2006).

15. Certain commercial equipment, instruments, or materials are identified in this paper to foster understanding. Such identification does not imply recommendation or endorsement by the National Institute of Standards and Technology, nor does it imply that the materials or equipment identified are necessarily the best available for the purpose.

16. See, for example, D. Cox, J. M. Key, M. S. Lai, J. R. Sutherland, M. R. Zahn, and J. M. Arrington, "On-line supercritical fluid extraction synchronous luminescence spectroscopy: a screening method for polycyclic aromatic hydrocarbons," J. Under. Chem. Res. 2, 39-42 (2005) and references therein.

17. See, for example, M. L. Lee, M. V. Novotny, and K. D. Bartle, Analytical Chemistry of Polycyclic Aromatic Compounds (Academic, 1981).

18. M. L. Lee, M. V. Novotny, and K. D. Bartle, Handbook of Polycyclic Aromatic Hydrocarbons, A. Bjørseth, ed. (Marcel Dekker, 1983).

19. N. J. Turro, Molecular Photochemistry (Benjamin/Cummings, 1965).

20. E. Clar, Polycyclic Hydrocarbons (Academic, 1964).

21. R. A. Friedel and M. Orchin, Ultraviolet Spectra of Aromatic Compounds (Wiley, 1951).

22. J. B. Birks, Photophysics of Aromatic Molecules (Wiley, 1970). 